\title{
Es gibt viel zu tun - wer packt es an?
}

Mit einigen Tagen Verspätung hat im November 2004 die neve EU-Kommission in Brüssel ihre Arbeit für die nächsten fünf Jahre aufgenommen. Für die neven Kommissare stehen eine ganze Reihe dringlicher umweltpolitischer Themen auf der

Agenda. An deren Bearbeitung wird die Kommission zeigen, ob sie Mut zu innovativem Denken und Handeln besitzt. Besonders wichtig sind die Klimaund die Chemikalienpolitik.

\begin{abstract}
A Von Nika Greger und Alice Wolfgramm ls Reaktion auf die Erweiterung und um die Verwirklichung zentraler politischer Prioritäten voranzutreiben, hat Kommissionspräsident José Manuel Barroso die bisherigen Ressorts leicht umstrukturiert und Kompetenzen verschoben. Getrennt wurden etwa die Bereiche Verkehr und Energie sowie Landwirtschaft und Fischerei. Ob und inwieweit Umweltpolitik in 2005 und darüber hinaus eine Rolle spielen wird, wird dabei immer mehr zu einer Schlüsselfrage in der EU und ihren Mitgliedstaaten.
\end{abstract}

\section{- Klima- und Chemikalienpolitik}

Konkret werden vor allem Maßnahmen gegen den Klimawandel im Mittelpunkt stehen. Auch wenn einige Staats- und Regierungschef gemeinsam mit den Chefs aus Wirtschaft und Industrie es gerne so sehen würden, mit dem In-Kraft-Treten des KiotoProtokolls im Februar sind die Gefahren des Klimawandels noch lange nicht gebannt. Vielmehr sollten 2005 endlich die nötigen Schritte angegangen werden, wie die EU in Zukunft ihre eigenen und die globalen Treibhausgase reduzieren will. Gelegenheiten dazu bieten sich einige, so findet im Mai ein erstes Regierungstreffen statt, auf dem es um globale Aktivitäten nach 2012 gehen soll. Im November findet dann das erste formelle Treffen der Kioto-Vertragsparteien statt. EU-intern wird die Kommission demnächst einen Vorschlag vorlegen, über den im Europäischen Rat im März diskutiert werden soll und auf dem sich die Europäer hoffentlich über eine detaillierte Strategie für die Zeit nach 2012 einigen. Der neue EU-Umweltkommissar Stravos Dimas aus Griechenland hat sich außerdem dafür ausgesprochen, dass künftig Emissionen aus dem Flugverkehr im Rahmen der Klimaverhandlungen mitdiskutiert werden sollen.

Ein weiterer großer Bereich wird neben dem Klimawandel die europäische Chemikalienpolitik REACH sein. Hier wird es nicht nur um die ei- gentliche Reform gehen, sondern auch darum, wie sich in Zukunft Umwelt- und Gesundheitsbelange gegenüber wirtschaftlichen Interessen behaupten werden. Interessant wird in diesem $\mathrm{Zu}$ sammenhang auch die weitere Entwicklung der so genannten extended impact assessments, mit denen die Auswirkungen einzelner Politikmaßnahmen ermittelt und vorausgesagt werden soll.

\section{- Lissabon-Strategie}

Von Präsident Barroso schon mehrfach angekündigt, wird einer der Hauptschwerpunkte der neuen EU-Kommission die Fortführung und Umsetzung der so genannten Lissabon-Strategie sein. Im März 2000 hatte der Europäische Rat auf seiner Tagung in Lissabon die Einleitung eines Maßnahmenkatalogs beschlossen, der die EU zum ,weltweit dynamischsten und wettbewerbsfähigsten Wirtschaftsraum" entwickeln soll. Nach der Theorie dieses auf zehn Jahre angelegten und seitdem als Lissabon-Strategie bezeichneten Prozesses treibt eine starke Wirtschaft die Schaffung von Arbeitsplätzen voran und fördert soziale und ökologische Maßnahmen, die wiederum eine nachhaltige Entwicklung und sozialen Zusammenhalt gewährleisten sollen.

Als kleines Stiefkind zu Lissabon wird sich die EU in diesem Jahr noch mit einer weiteren ihrer Strategien auseinandersetzen müssen. Eine Überprüfung der EU-Nachhaltigkeitsstrategie steht an, die Staats- und Regierungschefs werden auf dem Frühjahrsrat darüber diskutieren. Abgeleitet aus dem Sechsten Umweltaktionsprogramm muss die EU im Jahr 2005 sieben thematische Strategien vorlegen: Schutz der Meeresumwelt, Schutz vor Luftverunreinigungen, nachhaltige Ressourcenverwendung und Abfallrecycling sollen noch vor dem Sommer erscheinen. Nach der Sommerpause stehen dann die Strategien für die Bereiche Schutz der Böden, umweltverträgliche Pestizidverwendung und Schutz der Städtischen Umwelt an.
Mitte des Jahres müssen sich die europäischen Staats- und Regierungschefs auf einen Rahmen für die EU-Ausgaben von 2007 bis 2013 einigen, aus Umweltsicht ist dabei vor allem die Finanzierung des Natura 2000-Netzwerks relevant, da das Ergebnis dieser Budgetverhandlungen auch die Überprüfung, Umsetzung und Weiterentwicklung der EU-Strategie über die biologische Vielfalt ebenfalls auf der Agenda im Jahr 2005 - beeinflussen werden.

Mit den ökonomischen und ökologischen Inhalten der Gemeinschaftspolitiken eng verknüpft sind die Auswirkungen der im vergangenen Jahr vollzogenen Erweiterung der Union. Es gibt erhebliche Defizite bei der Umsetzung des europäischen Umweltrechts, dessen Erfüllung besonders in den neuen Mitgliedstaaten sichergestellt werden muss. Die Europäische Gemeinschaft hat in den nächsten Jahren große Integrationsleistungen nicht nur auf wirtschaftlicher, sondern auch auf gesellschaftspolitischer Ebene zu vollbringen, die die Kommission nach Kräften unterstützen und vorantreiben muss.

\section{- Intensivierung der Umweltpolitik}

Auf der neuen Kommission lastet in den kommenden Jahren ein enormes Arbeitspensum. Sie sollte im Rahmen des integrierten Ansatzes in der Wirtschafts- und Umweltpolitik ihre Bemühungen vor allem in den Bereichen Klimaschutz, Chemikalienpolitik und Biodiversität verstärken.

Die kommenden fünf Jahre werden außerdem zeigen, inwieweit die neue Kommission auf altmodische und auslaufende Wirtschaftsmodelle und ihre Verfechter in den Wirtschaftsverbänden setzen oder sich auf neue und innovative Wirtschaftsbereiche konzentrieren, die erkannt haben, dass ohne Umwelt- und Ressourcenschutz Wettbewerbsfähigkeit nicht zu haben ist. Business as usual-Szenarien, die auf die Ausbeutung der natürlichen Ressourcen und die immer weitere Aufweichung sozialer und ökologischer Rechte setzen, werden die Wirtschaftsfähigkeit der EU wohl kaum stärken.

\section{Die Autorinnen}

Nika Greger ist Leiterin der Berliner Geschäftsstelle des Deutschen Naturschutzrings (DNR). Alice Wolfgramm ist dort Mitarbeiterin im Bereich EU-Koordination.

Kontakt: DNR, Prenzlauer Allee 230, 10405 Berlin. Tel. 030-44339181, E-Mail: nika.greger@dnr.de 
(c) 20I0 Authors; licensee IÖW and oekom verlag. This is an article distributed under the terms of the Creative Commons Attribution Non-Commercial No Derivates License (http://creativecommons.org/licenses/by-nc-nd/3.o/), which permits unrestricted use, distribution, and reproduction in any medium, provided the original work is properly cited. 